\title{
Prevalência de cárie em crianças do ensino fundamental de Umuarama, Paraná
}

\author{
Caries prevalence in children of elementary school of Umuarama, Paraná
}

Prevalencia de caries em niños de la enseñanza primaria de escuelas municipais de Umuarama, Paraná Andressa Juliana Martins DEMEU'

Larissa Pinheiro VIUDES ${ }^{1}$

Leonardo Carlini BARBOSA ${ }^{1}$

Vanessa Rodrigues do NASCIMENTO2

${ }^{1}$ Acadêmica de Odontologia pela Universidade Paranaense - UNIPAR - Umuarama/PR

${ }^{2}$ Docente do Curso de Odontologia da Universidade Paranaense - UNIPAR - Umuarama/PR

\section{Resumo}

Os levantamentos epidemiológicos são de extrema importância para o conhecimento da prevalência da doença cárie, que revelam a realidade social, epidemiológica e principalmente o estado de saúde atual da população estudada e a eficácia dos serviços de saúde de um determinado local. Este trabalho teve por objetivo analisar a prevalência de cárie para a população de escolares do ensino fundamental da rede municipal de Umuarama /Paraná. Foram examinados 2.350 escolares com a faixa entre 6 e 11 anos. As faixas etárias foram divididas em dois grupos: 6-8 anos, a média CPO-D para este grupo foi 0,25. Com relação à faixa etária de 9 a 11 anos foi observado uma média para o CPO-D 0,6. Para a realização do ceo-d foram examinadas 193 escolares com a faixa etária de 5 anos e a média de ceo-d para este grupo foi de 1,08. Concluindo que a classificação da prevalência de cárie para o município de Umuarama, seguindo os parâmetros da OMS (1982), baseada na média do índice CPO-D para a faixa etária estudada foi classificada como muito baixa $(0,0$ a 1,1$)$ e a média para o índice ceo-d foi considerada baixa $(1,08)$.

Descritores: Cárie Dentária; Criança; Epidemiologia.

\section{Abstract}

The epidemiological surveys are of the extreme attention to awareness of the prevalence of the dental caries diseases, which reveal the social reality and epidemiological mainly the current mental health of the population studied and efficiency of the health services of a particular place. This abstract seek analyze the prevalence of dental caries of the school population of the elementary school of the municipal network of Umuarama/Paraná. 2.350 schools were analyzed with age range between 6-11 years old. The age range were divided in two groups : 5 with average CPO-D for this group were 0,25. With regard to age range of 9-11 years old were observed an average for the CPO-D 0,6. In short the classification from prevalence dental caries for the municipality of Umuarama, according to parameters of the OMS ( 1982) based in average of the index CPO-D were rated as very small $(0,0$ a 1,1) and the average from the index CEO-D were considered.

Descriptors: Dental Caries; Child; Epidemiology.

\section{Resumen}

Los levantamientos epidemiológicos son de extrema importancia para el conocimiento de la prevalencia de la enfermedad caries, que relevan la realidad social y epidemiológica, principalmente el estado de salud actual de la población estudiada, y la eficacia de los servicios de salud de uno determinado lugar. Este trabajo tuvo por objetivo analizar la prevalencia de caries en la población de escolares de la enseñanza de la red de Umuarama/Paraná. Se examinaron 2.350 escolares con las edades entre 6 y 11 años. Los grupos de edad se dividieron en tres: 5 años con media de ceo-d de 1,08, y 6-8 años con media de CPO-D de 0,25. Con relación al grupo de edade de 9-11 años, fue observada una media para el CPO-D de 0,6. Concluyendo que la clasificación de la prevalencia de caries para el municipio de Umuarama, siguiendo los parámetros de la OMS (1982), basado en la media del índice CPO-D fui clasificado como muy baja (0,0 hasta 1,1), e la media para el índice ceo-d fue considerada muy baja.

Descriptores: Caries Dental; Niño; Epidemiologia.

\section{INTRODUÇÃO}

O modelo assistencial de saúde do Brasil passa por um processo de mudança em suas ações, onde estas passaram de uma qualificação de atendimento na sua grande maioria curativa para uma abordagem voltada para ações preventivas, tendo como ponto central o indivíduo e os determinantes sociais de saúde ${ }^{1-3}$.

As ações de Saúde Bucal no Sistema Único de Saúde (SUS) eram anteriormente ofertadas de forma paralela ao processo de organização dos demais serviços de saúde, com baixo poder de resolubilidade, sendo incapazes de equacionar os principais problemas da população ${ }^{1-3}$.

Na última década do século XX e na primeira do século XXI, foram fortemente implantadas e expandidas importantes intervenções em saúde bucal em todo o país, tendo como referência o princípio constitucional da universalização das ações e serviços de saúde, a exemplo da fluoretação das águas de abastecimento público, do atendimento odontológico da rede pública do SUS e da adição de compostos fluorados aos dentifrícios. É nesse sentido que, de forma clara, observa-se uma tendência de redução da prevalência e severidade da cárie dentária no Brasil, consistente também com o controle da doença em grande parte do mundo ocidental ${ }^{1-3}$.

Na Odontologia a cárie dentária é a doença de maior prevalência na cavidade bucal, sendo considerada como um problema de saúde pública pois trata-se de uma doença que abrange ainda uma quantidade alta de pessoas, causando morbidade e mutilação nos indivíduos acometidos. Apresenta uma etiologia multifatorial e que ocorre quando há um desequilíbrio do processo dinâmico entre a estrutura dentária e o seu meio ambiente, sendo necessária a interação de três fatores como substrato cariogênico (dieta), microbiota bucal cariogênica e características do hospedeiro que compreendem morfologia dental, constituição e fluxo salivar susceptível para desencadear esse processo. Esta concepção da doença como multifatorial nos remete que para promover a saúde bucal das populações, é necessário levar em conta as variáveis biológicas, os fatores sociais e psicológicos, pois ter saúde não é somente uma escolha, mas uma condição determinada pelo meio social, cultural e econômico ${ }^{3,5-6}$. 
Os levantamentos epidemiológicos são de extrema importância para o conhecimento da prevalência da doença cárie, pois revelam a realidade social e epidemiológica e principalmente o estado de saúde atual da população estudada e a eficácia dos serviços de saúde de um determinado local, assim como é possível através de seus resultados realizar planejamentos de ações de promoção de saúde bucal em todos os níveis de prevenção, para a eleição de grupos e demandas prioritários de atenção ${ }^{7-18}$.

Este trabalho teve por objetivo analisar a prevalência de cárie da população de escolares do ensino fundamental e educação infantil das escolas municipais de Umuarama/ Paraná utilizando o índice CPO-D no ano de 2016 e ceo-d no ano de 2018 respectivamente.

\section{MATERIAL E MÉTODO}

Este estudo tratou-se de uma pesquisa A realização dessa pesquisa obedeceu aos preceitos dentro dos padrões determinados pelo Comitê de Ética em Pesquisa em Seres Humanos da Universidade Paranaense e está registrada com o parecer de número 2.338.309.

A cidade de Umuarama está situada na região noroeste do Estado do Paraná, região Sul do Brasil. Possuía uma população em 2010 de 100.676 mil habitantes e com estimativa para 2017 de 109.955 mil, segundo os dados do Instituto Brasileiro de Geografia e Estatística (IBGE).

Inicialmente foi realizada uma parceria entre a Universidade Paranaense (UNIPAR) - Unidade de Umuarama e a Secretaria de Educação do município de Umuarama, em nome das escolas públicas, e Secretaria Municipal de Saúde - Departamento Odontologia, para que se pudesse realizar a pesquisa nos estabelecimentos de ensino, que totalizam 22 escolas municipais com aproximadamente 5.165 escolares na faixa etária de 5 a 11 anos.

Este estudo trata-se de um estudo do tipo transversal e de caráter descritivo quantitativo, obtendo amostra por conveniência e a coleta de dados aconteceu de julho a dezembro de 2016 para o índice CPO-D e serviu como referência para as estratégias adotadas para a disciplina de Saúde Coletiva e de janeiro a agosto de 2018 para o índice ceo-d, realizadas também na mesma disciplina.

O cálculo do tamanho da amostra para a realização do índice CPO-D foi feito pelo programa Epi-Info, de domínio público, o qual possibilita o cálculo de tamanho mínimo da amostra para estabelecimento de prevalência em populações finitas, utilizando-se nível de confiança de $95 \%$. O número total de crianças na faixa etária de 6 a 11 anos determinadas para inclusão foi de 5165, totalizando 1.086 crianças que compuseram a amostra final, porém foram incluídas 2.350.

Para a realização do índice ceo-d, foram incluídas no estudo as escolas municipais que possuem educação infantil e dentre estas as quais não possuem assistência odontológica municipal in loco. Desta forma totalizaram-se 5 escolas das 16 que possuem alunos em idade pré-escolar, 263 alunos aproximadamente através da estimativa da Secretaria Municipal de Educação.

Foi adotado como critério de inclusão que os responsáveis legais pelos escolares aceitassem a realização do exame através da assinatura do Termo de Consentimento Livre Esclarecido (TCLE), disponibilizado pela UNIPAR e distribuído pelas escolas. Foi excluído da pesquisa o escolar que o responsável não concordou com o exposto no Termo de Consentimento Livre Esclarecido, não compareceu na escola no dia marcado para a coleta de dados ou ainda não permitiu a avaliação.

Os exames clínicos foram realizados por professores do Curso de Odontologia da UNIPAR Umuarama da disciplina de Saúde Coletiva II, auxiliados por acadêmicos do terceiro e quarto ano do curso já citado. Estes participantes dividiram as funções de examinadores em número de 4 , anotadores também somando 4 e coordenadores que foram os professores da disciplina.

Para minimizar as variações entre os diferentes examinadores, assegurando uma interpretação uniforme e consistente dos critérios padronizados para a coleta dos dados, foram realizados exercícios de calibração com os estudantes e professores denominados como observadores e anotadores, in loco, durante outras atividades semelhantes, da disciplina de Saúde Coletiva II no ano anterior a este levantamento epidemiológico.

Previamente a realização dos exames intra bucais, foi preconizada a escovação supervisionada dos alunos avaliados para que fosse conseguida uma interpretação correta das superfícies dentais. As avaliações foram realizadas nas escolas municipais de Umuarama, em locais designados pela coordenação da escola (sala de aula ou pátios), dotados de boa iluminação artificial ou natural. Cada equipe foi composta de um coordenador, acadêmico observador e anotador, utilizou-se de espátulas de madeira, lanterna e os EPI's (gorro, jaleco, máscara e luvas) e os exames transcorreram respeitando-se as normas de biossegurança.

Para os escolares matriculados no ensino fundamental foi utilizado o índice CPO-D, para os matriculados na educação infantil utilizou-se o ceo-d ambos com a função de medir e comparar a experiência de cárie dentária, sendo que seu valor expressa a média de dentes cariados, perdidos e obturados em um indivíduo e/ou grupo de indivíduos em dentes permanentes e decíduos respectivamente.

As informações obtidas no exame para escolares matriculados no ensino fundamental foram anotadas em um formulário de CPO-D, ou ceo-d individual (Figura 1), a identidade do indivíduo 
escolar foi mantida em sigilo, assim como, a comunicação entre observador e anotador era realizada através de códigos.

Os critérios adotados neste estudo tomaram por base a $4^{a}$ edição do Oral Health Surveys - Basic Methods da Organização Mundial da Saúde, descritos no Manual do Examinador e no Manual do Anotador, produzidos pela equipe de coordenação do Projeto SB Brasil.

Após a realização dos exames e preenchimento das fichas, os dados foram tabulados em uma planilha de excell e submetidos a uma análise descritiva.

Os escolares que apresentavam necessidade de tratamento eram encaminhados para atendimento na Clínica Odontológica da UNIPAR, os responsáveis e a direção escolar eram avisados.

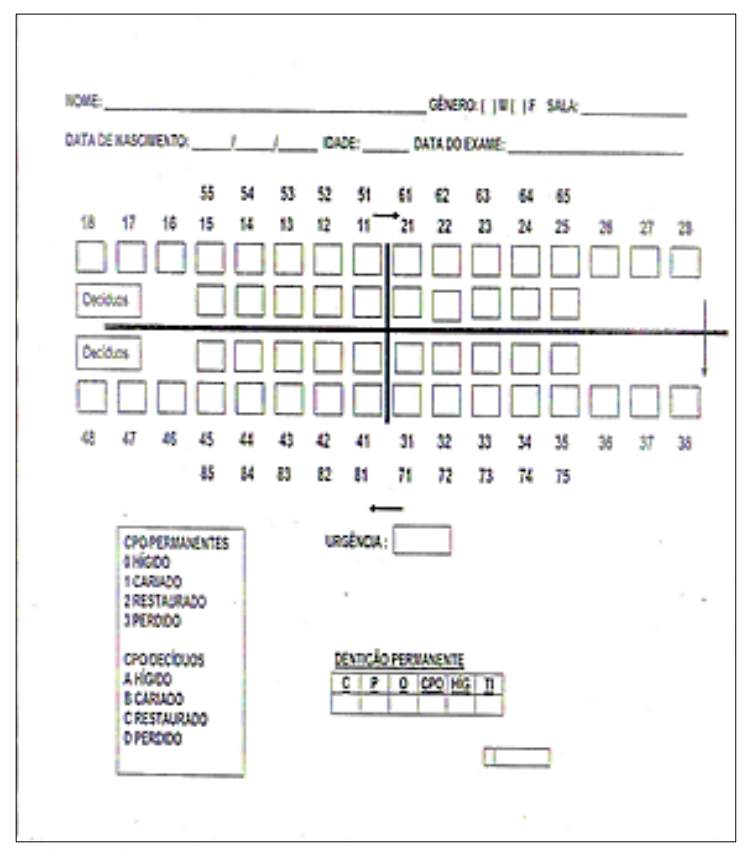

Figura 1: Formulário para anotação das informações após exame.

\section{RESULTADOS}

Para a realização do CPO-D foram examinados 2.350 escolares do ensino fundamental municipal de Umuarama - Paraná, com a faixa etária entre 6 e 11 anos. O primeiro grupo, composto por crianças de 6-8 anos , a média CPO- D para este grupo foi 0,25 . Os componentes "cariado", "obturado" e perdido representaram respectivamente $2,55 \%$ (324), $1,5 \%$ (197) e $0,06 \%$ (8) enquanto que os hígidos representaram 95,8\%(12.146) do total de dentes examinados (12.675). Com relação a faixa etária de 9 a 11 anos (totalizando uma amostra de 1.272) foi observado uma média para o CPO-D 0,6. Os componentes "cariado", "obturado" e perdido representaram respectivamente $3,21 \%$ (593), $0,92 \%$ (171) e $0,04 \%$ (8) enquanto que os hígidos representaram 95,8\% (17.646) do total de dentes examinados (18418).

Para a realização do ceo-d foram examinadas 193 escolares com a faixa etária de 5 anos de idade matriculados na educação infantil municipal de Umuarama - Paraná. A média de ceo-d para este grupo foi de 1,08. Os componentes "cariado", "extração indicada" e "obturados" representaram respectivamente: $4,80 \%$ (173), $0,11 \%(4)$ e $0,60 \%(22)$ enquanto os hígidos representaram $94.49 \%$ (3424) do total de dentes examinados (3623)

A classificação da prevalência de cárie para o município de Umuarama, seguindo os parâmetros da OMS (1982), baseada na média do índice CPO-D foi classificada como muito baixa $(0,0$ a 1,1$)$ e para o ceo-d foi classificada como baixa $(1,08)$

\section{DISCUSSÃO}

A prevalência da doença cárie é avaliada nas populações por meio de levantamentos epidemiológicos, que revelam principalmente o estado de saúde atual da população estudada e a eficácia dos serviços de saúde de um determinado local, também permitindo o diagnóstico precoce de lesões tornando o tratamento mais simples, menos invasivo e com menor custo, envolvendo ações de promoção em saúde com o objetivo de mudanças de comportamento com relação à dieta e à higiene oral. Dessa forma, os resultados encontrados a partir desses levantamentos podem ser utilizados para direcionar e avaliar os serviços odontológicos para uma determinada população ${ }^{1-7}$.

$\mathrm{O}$ mais recente levantamento epidemiológico de saúde bucal nacional foi o SB Brasil 2010, no qual participaram indivíduos residentes em 177 municípios das cinco macrorregiões. Foram realizados exames bucais para avaliar as condições de saúde e os principais agravos e utilizou-se de questionários para coleta de dados sobre a condição socioeconômica, acesso aos serviços odontológicos e percepção de saúde.

Apesar dos quatro levantamentos epidemiológicos nacionais (1986, 1996, 2003 e 2010) e dos inúmeros realizados até hoje em diversos municípios brasileiros mostrarem mudanças na prevalência de cárie da população, ainda se tem uma grande necessidade de avaliar a saúde bucal brasileira. Esta necessidade se explica devido aos perfis divergentes apresentados pelas macrorregiões ${ }^{6}$.

Neste estudo realizado em escolares do ensino fundamental da rede municipal de Umuarama no Paraná, utilizou- se uma faixa etária diferente da preconizada pela Organização Mundial de Saúde para o CPO-D que seria dos 12 anos; nesta pesquisa utilizamos a faixa etária de 6-9 anos, sendo avaliados apenas os dentes permanentes nos casos onde o escolar apresentava dentição mista, isto para que fosse possível realizar uma comparação mais fiel com o principal e mais recente levantamento epidemiológico realizado no Brasil (SB Brasil). Os autores também apontam a importância em se reconhecer a situação real da doença cárie em todas 
as faixas etárias para que seja possível a avaliação dos métodos preventivos e curativos adotados pelo local assim como traçar novas estratégias de prevenção da cárie dentária na segunda infância, já que este procedimento requer ações amplas e multidisciplinares, visto que a etiologia desta doença é complexa, sendo necessária uma ação multidirecional.

A média para o CPO-D de escolares municipais, na faixa etária entre 6-11 anos da cidade de Umuarama foi 0,42 sendo mais favorável do que o encontrado no SB Brasil, cuja média do índice CPOD aos 12 anos em 2010 foi de 1,53 para a cidade de Curitiba, capital do Paraná e de 1,66 para a região Sul, os resultados da média do ceo-d para escolares da educação infantil foi de 1,08 , também mais favorável do que o último levantamento nacional em 2010 que foi de 1,97 para a região Sul e Curitiba 1,92. Estes resultados representam também o alcance da meta proposta pela OMS de CPO-D menor que 3 nas crianças de 12 anos, permitindo estabelecer que a doença cárie pode ser considerada controlada no município, esta comparação é realizada com cuidado pois a faixa etária utilizada entre os dois levantamento é diferente e o aumento dos índices de dentes cariados, perdidos e obturados (CPO-D) proporcionalmente ao aumento da idade, isto foi também observado neste levantamento pois quando a amostra foi dividida em faixa etária os escolares com idade entre 6 a 8 anos apresentaram uma media do índice CPO-D menor $(0,25)$ quando comparado com a faixa etária entre 9 a 11 anos $(0,60)^{11}$.

O impacto de programas de saúde bucal direcionados para crianças em idade escolar tem sido identificado nos levantamentos conduzidos pelo Ministério da Saúde como protagonistas, ao revelarem a redução significativa nos índices de cárie em dentes permanentes. Isto nos leva a afirmar que alguns fatores como ações de promoção de saúde em todos os níveis de prevenção englobando a incorporação de íons flúor nas águas de abastecimento, melhorias das condições de vida e na saúde pública, assim como as mudanças na ciência e tecnologia para a detecção e diagnóstico dessa doença possibilitaram a redução desse índice, auxiliando também no melhor conhecimento da história natural da doença cárie ${ }^{2-6}$.

A realização de estudos epidemiológicos é necessária, porém existem barreiras durante sua realização, que iniciam com a dificuldade em receber os TCLE de volta, assinado pelos pais, e continuam com a ausência dos escolares no dia da realização do exame e ainda com a mudança na rotina da escola visitada para o levantamento, esses pontos foram encontrados neste estudo, onde foram visitados as 22 escolas municipais de Umuarama. Esta situação reforça a influência dos determinantes sociais de saúde na condição de saúde bucal na população, onde foi verificado que as crianças que mais precisavam de cuidados eram aquelas em que não possuíam os termos ou era percebido um descuido dos responsáveis. CONCLUSÃO

O índice CPO-D para os escolares com faixa etária entre 6 a 8 anos matriculados nas escolas municipais de Umuarama/Paraná foi de 0,25, classificado como muito baixo pela OMS.

O índice CPO-D para os escolares com faixa etária entre 9 a 11 anos matriculados nas escolas municipais de Umuarama/Paraná foi de 0,6, considerado como muito baixo pela OMS.

$O$ índice ceo-d para os escolares com faixa etária de 5 anos nas escolas municipais de Umuarama/Paraná foi de 1,08, considerado como baixo pela OMS.

\section{REFERÊNCIAS}

1. Narvai PC. Cárie dentária e flúor: uma relação do século XX. Ciênc saúde colet. 2000;5(2):381-92.

2. Cortelli SC, Cortelli JR, Prado JS, Aquino DR, Jorge AOC. Fatores de risco a cárie e CPOD em crianças com idade escolar. Brasil. Ciênc Odontol Bras. 2004;7(2):75-82.

3. Mialhe FL, Melo MM, Berti M, Dobrowolski M. Contribuição das ações de educação em saúde da disciplina de odontologia em saúde coletiva na consolidação das DCN e do SUS: relato de uma experiência. Arq Ciênc Saúde Unipar. 2007; 11(3):193-97.

4. Baldani MH, Vasconcelos AGG, Antunes JLF. Associação do índice CPO-D com indicadores sócio-econômicos e de provisão de serviços odontológicos no Estado do Paraná, Brasil. Cad Saúde Pública. 2004;20(1):143-52

5. Baldani MH, Antunes JLF, Pupo YM, Lawder JAC, Silva FFM. Determinantes individuais da utilização recente de serviços odontológicos por adolescentes e adultos jovens de baixa renda. Pesq Bras Odontoped Clin Integr. 2011;11(1):91-8.

6. Ministério da Saúde. Brasil: Coordenação Nacional de Saúde Bucal. Condições de Saúde Bucal Da População Brasileira - Projeto SB Brasil 2003 - [Citado 2010 dez.]. Disponível em: HTTP://portal,saude.gov.br/saude/arquivos/pdf/rel atorio_brasil_sorridente.pdf.

7. Geus JL, Luca CMB, Baldani MH, Czlusniak GD. Prevalência de Cárie e Autopercepção da Condição de Saúde Bucal entre Crianças de Escolas Urbanas e Rurais de Ponta Grossa - PR. Pesq Bras Odontoped Clin Integr. 2013;13(1):111-17.

8. Lazzarin HC, Sulzler KE, Kramer IV, Camargo JB. Prevalência de cárie dentária em crianças de uma instituição pública do município de Cascavel - PR, Brasil. Arquivos do MUDI. 2016;20(3):13-24.

9. Vicente VA, Polleto MM, Neiva IF, Pinto JVT, Braga SF, Moreira $M$ et al. Relação entre a 
prevalência da doença cárie e risco microbiológico. Cienc Odontol Bras 2008;11(2):44-8.

10.Porcelli ICS, Partyka J, Matsuura E, Fracasso MLC, Maciel SM. Prevalência e Associação Entre Cárie Dentária e Má Oclusão em Adolescentes de Londrina, Paraná, Brasil. UNICIÊNCIAS. 2015;19(2):106-11.

11.Baltazara MMM, Giordania MT, Furlanettoa DLC, Bertia M, Bianchia FJ. Levantamento das Condições de Saúde Bucal de Crianças e Adolescentes das Comunidades Rurais Atendidas em Unidade Móvel de Saúde no Oeste do Paraná. UNOPAR Cient Ciênc Biol Saúde. 2011;13(4):257-61.

12. Amaral MA, Ruiz DT, Simm W, Pereira JS, Luiz MAF, Matsuo T. Desgaste Dentario: Estudo Epidemiológico em uma População de Cinco Anos de Sarandi, Paraná. Saúde e Pesquisa. 2008;1(2):151-56.

13. Caldas C, Mialhe F, Silva R. Prevalência de manchas dentais extrínsecas negras e sua relação com a cárie dentária em crianças do município de Santa Terezinha de Itaipu - PR. RFO. 2008;13(2):22-6.

14. Vicente VA, Polleto MM, Neiva IF, Pinto JVT, Braga SF, Moreira M. Relação entre a prevalência da doença cárie e risco microbiológico. Cienc Odontol Bras. 2008;11(2):44-8.

15.Dubinski P, Cardoso SA, Hoeppner MG. Avaliação das Causas das Substituições de Restaurações nas Disciplinas de Dentística II e Clínica Integrada do Curso de Odontologia da UNIPAR - Campus Umuarama. UEPG Ci Biol Saúde. 2005;11(1):7-14.

16.Berti M, Furlanetto DLC, Walker MMS, Baltazar MMM, Bianchi FJ. Levantamento epidemiológico de cárie dentária em escolares de 5 e 12 anos de idade do município de Cascavel, PR. Cad Saúde Colet. 2013;21(4):403-6.

17. Castillo LO, Moro RC, Zanatta FB, Flores DM. Prevalência de cárie dentária nos alunos da Escola Municipal Adelmo Simas Genro, Santa Maria, RS: uma análise descritiva parcial. Dis Scientia. 2016;7(1):121-25

18.Cangussu MCT, Lopes LS. Epidemiologia da Cárie Dentária no Estado da Bahia de 1986 a 2012. Rev baiana saúde pública. 2012;36(3):640-50.
CONFLITO DE INTERESSES

Os autores declaram não haver conflitos de interesse.

AUTOR PARA CORRESPONDENCIA

Vanessa Rodrigues do Nascimento

vanessanascimento@prof.unipar.br

Submetido em 07/12/2018

Aceito em 12/03/2019 